\title{
Practical Perceptually Adaptive Approach to Video Logo Placement in TV Broadcasts
}

\author{
Alexander Wong \\ Department of Electrical and Computer Engineering \\ University of Waterloo \\ Waterloo, Ontario, Canada, N2L 3G1 \\ Email: a28wong@engmail.uwaterloo.ca
}

\author{
William Bishop \\ Department of Electrical and Computer Engineering \\ University of Waterloo \\ Waterloo, Ontario, Canada, N2L 3G1 \\ Email:wdbishop@uwaterloo.ca
}

\begin{abstract}
A common technique used by broadcast stations for video copyright protection is video logo placement, where a logo indicating ownership is inserted into the video broadcast in a visible way. This paper presents a practical algorithm that performs adaptive video logo placement in TV broadcasts based on a perceptual model from both spatial and temporal perspectives. The algorithm adjusts the position and the transparency of the video logo based on content activity, inter-frame relationships, and contrast with the goal of reducing overall perceptual loss while maintaining logo visibility. The algorithm is computationally efficient and suitable for video logo placement in live broadcasts. Experimental results show that the adaptive video logo placement algorithm achieves a good balance between perceptual quality and logo clarity.
\end{abstract}

\section{INTRODUCTION}

Digital video broadcasting and distribution has highlighted the importance of copyright protection for digital video content. An effective method used for indicating the ownership of video and image content is digital watermarking where additional information is embedded into a video or image. For copyright protection, the embedded information indicates the ownership of the digital media. For example, a corporate logo could be used to indicate ownership. Digital video and image watermarking can typically be divided into two categories: (i) Invisible watermarking [1]-[6], and (ii) Visible watermarking [7]-[12]. An overview of video watermarking techniques can be found in [13].

Invisible watermarking embeds information into the video or image content such that is not visible to the human eye. The main advantage of invisible watermarks is that they have a negligible effect on perceptual quality. However, invisible watermarks require special hardware or software to detect the watermark. Furthermore, such techniques are typically less robust to content manipulation. Common uses of invisible watermarks include content authentication and traitor tracing.

Visible watermarking embeds information into the video or image content such that it is visible to the human eye. Unlike invisible watermarking, this technique reduces perceptual quality. Common uses of visible watermarks include ownership identification and marking to deter unauthorized use. Most video watermarking research has focused on invisible video watermarking for compressed video content. Visible watermarking is less often the subject of research.
One common approach used by broadcasting stations for video copyright protection is video logo placement, a form of visible watermarking where the logo of the owner is placed into a video broadcast in a visible manner. In the case of TV broadcast stations, the logo of the TV station is inserted into TV broadcasts. Video logo placement is widely used for TV broadcasts for a number of reasons. First, many TV broadcasts are live broadcasts, where video is delivered in "real-time" to the audience. Examples of live broadcasts include sporting events, award shows, and talk shows. The simplicity of video logo placement allows a TV broadcasting station to embed the station's logo into the live broadcast stream with little processing time. Furthermore, video logos provide a very clear and explicit indication of video content ownership without covering a large area of the video broadcast content.

Currently, video logos are positioned in a fixed location in all video frames of a TV video broadcast. There are several problems associated with placing a video logo at a fixed location in all video frames of a video broadcast. First, it is easy for someone to remove the video logo with little loss in video quality by cropping all video frames at a fixed location. Another problem is that the fixed logo placement may obstruct important video content that occurs at the fixed location. This leads to a loss in perceptual quality. One approach to reducing the perceptual quality loss is the use of semi-transparent video logos, such that the underlying video broadcast content can be partially viewed. However, the use of logos at a fixed transparency level for all frames may lead to significantly reduced logo clarity in some situations.

This paper attempts to address all of the forementioned issues using a perceptual model, with the primary focus on retaining perceptual quality in the video broadcast after the placement of the video logo. While other adaptive video and image watermarking techniques have utilized perceptual models [2], [4]-[6], [10], [12], they are fundamentally different than the proposed algorithm. First, several of the previously researched techniques have been designed for the purpose of invisible watermarking [2], [4]-[6]. Of the techniques designed for visible watermarking [10], [12], none of them consider the perceptual quality and security implications of watermarking from a temporal perspective, which is important for video content. The proposed algorithm takes both spatial and tem- 
poral aspects of perception into consideration. Furthermore, the proposed algorithm is designed specifically around the requirements of TV broadcasts.

In this paper, the proposed algorithm is described and explained in detail in Section 2. The experimental results used to demonstrate the effectiveness of the proposed algorithm are presented in Section 3. Finally, conclusions are drawn in Section 4.

\section{Proposed Algorithm}

Four criteria must be considered carefully when designing a video logo placement algorithm for TV video broadcasts. First, the video logo should be positioned such that it avoids obscuring important content that occurs in the video. Second, the video logo should be visible at all times in the video. Third, the video logo should not be easily removable. Finally, for live broadcasts, the video logo placement process should not result in significant computational overhead.

Based on these four criteria, the proposed algorithm is divided into two main processes: i) video logo positioning, and ii) video logo transparency adjustment. The adaptive logo positioning process is designed to satisfy the first and third criterion, while the adaptive logo transparency adjustment is designed to satisfy the second criterion. Furthermore, the entire algorithm framework should be designed with efficiency in mind to satisfy the final criterion.

\section{A. Logo Positioning}

In the proposed algorithm, the logo is adaptively positioned in a video broadcast stream based on two main factors: i) perceptual content activity, and ii) inter-frame relationships. The first main factor under consideration is perceptual content activity. Currently, video logos are placed at fixed locations within all video frames. As such, perceptually important content may be concealed by the video logo if it appears at the fixed location. To avoid obscuring perceptually important content that occurs in the video broadcast, the logo should be placed at a location where there is little to no perceptual content activity. Therefore, by measuring the perceptual content activity at various locations of the video broadcast, the video logo may be placed at a location with low perceptual content activity to reduce the perceptual loss caused by the video logo. A secondary benefit is that it increases the difficulty of removing the video logo from the video.

The second main factor under consideration in the proposed algorithm is inter-frame relationships. Video frames exhibit two different types of inter-frame relationships. From a visual perspective, consecutive video frames often appear similar to each other in terms of visual content. From a semantic perspective, a sequence of video frames may be grouped together to indicate a sequence of related frames even if the frames do not exhibit visual similarities. For example, a sequence of frames captured within a continuous period of time by a single camera could represent a single shot. These inter-frame relationships can be exploited to improve video logo placement from both a perceptual quality and security perspective.

Dynamic visible watermarking algorithms operate on a frame-by-frame basis. For example, a random transform may be applied to the video logo for each frame [11]. The position of the video logo may also change from frame to frame [12]. Both of these approaches can distract the viewer. As such, most current systems place video logos at fixed positions. Static logo placement is less distracting. However, such approaches fare poorly in terms of copyright protection, as video logos can be easily removed by cropping the video in a fixed manner. The issues associated with the dynamic and static video logo placement algorithms can be addressed by exploiting inter-frame relationships. By fixing the position of the video logo for a sequence of related frames while encouraging position change for different sequences, perceptual quality loss can be significantly reduced while improving video logo protection. Furthermore, the process of evaluating perceptual content activity can be improved by making use of statistics from related frames to better reflect the video content. Therefore, by taking advantage of perceptual content activity and inter-frame relationships, the proposed algorithm takes into account both the spatial and temporal characteristics of the video broadcast.

In the proposed algorithm, the video broadcast content is first segmented into individual shots. This is achieved differently depending on the type of video broadcast being processed. For pre-recorded video broadcasts, the shot boundaries in the broadcast stream can be found either manually or through the use of a shot boundary detection algorithm. In the case of a live video broadcast, shot boundaries are determined in real-time either based on known camera shot transitions or through the use of a dynamic shot boundary algorithm that uses previous and current video frames. Shot segmentation is needed as the proposed algorithm determines the position of the video logo on a shot-by-shot basis. By allowing for positional changes only when changing shots, the level of distraction experienced by the audience is kept to a reasonably low level.

To determine the position for placing the video logo into the video frames within a shot, the first $k$ frames within the shot are used to acquire local perceptual activity statistics. In the case of a live broadcast, the first 5 frames within a shot may be used to acquire local activity statistics since further frames may not be available. An example of this is illustrated in Fig. 1. By making use of information from multiple video frames, a better overall picture of local perceptual content activity statistics within the shot can be obtained to help improve logo placement. For prerecorded video broadcasts, more video frames from the shot can be used to provide better activity statistics.

Once a set of $k$ video frames have been selected from the shot, it is important to consider the candidate regions where the video logo can be placed within the context of video broadcasts. This is contrary to a general watermarking algorithm [12], which may place a watermark at any location 


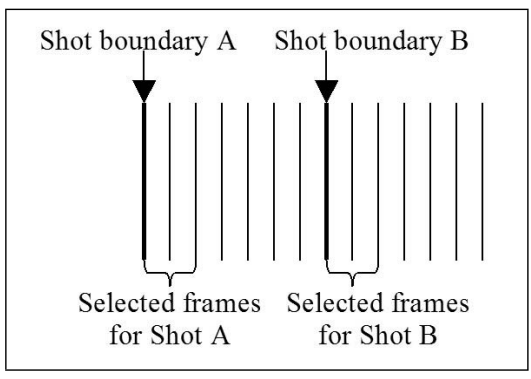

Fig. 1. Sample Frame selection process

(such as the centre) of the image depending on the underlying content. First, video logos should be placed away from the central area of the broadcast video content. Furthermore, the rule of thirds can be used to avoid drawing excessive attention to the video logo. Imagine that a frame is divided into thirds by two horizontal lines and two vertical lines. The rule suggests that elements of interest should be placed at the intersecting points between the horizontal and vertical lines. As such, it is important to place the video logo away from these points to avoid unnecessary focus on the logo. Therefore, the candidate regions used in the proposed algorithm are limited to the regions near the four corners of the video frame, each $25 \%$ of the width and height of the frame. The candidate regions in each video frame are then divided into $n$ blocks. An example of this process is illustrated in Fig. 2.

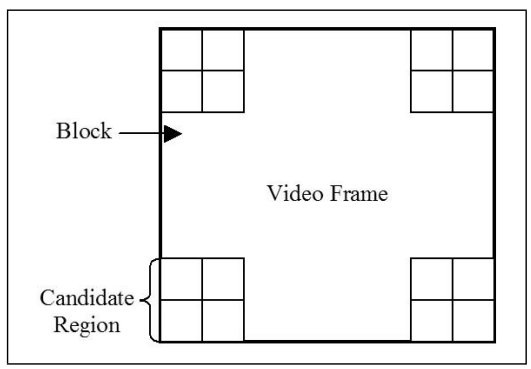

Fig. 2. Candidate region partitioning scheme

The size of each block is set less than or equal to the size of the video logo. The overall perceptual content activity level within the $i$ th block is calculated as the sum of spatial variances within the $i$ th block of each video frame from the set under evaluation:

$$
A c t_{i}=\sum_{j=1}^{k} \operatorname{var}_{j}(i)
$$

where $A c t_{i}$ is the perceptual content activity level of the $i$ th block, $k$ is the total number of video frames selected for evaluation, and $\operatorname{var}_{j}(i)$ is the spatial variance of pixel intensities within the $i$ th block in the $j$ th selected video frame. The spatial variance is chosen as an activity metric since it has a low computational complexity and it provides a good representation of visual activity. This makes it suitable for live broadcasts with real-time video logo placement requirements.
To encourage the position change of a video logo over time, a block-based usage counter system is used. Each block in a video frame is assigned a counter, which increments and decrements based on video logo position usage. At the beginning of a video broadcast, all counters are set to zero. When the position of the video logo is determined at a particular shot, the counters are adjusted based on whether the video logo covers a particular block. If a block is covered by the video logo, the counter for that block is set to a fixed value $\beta$. For testing purposes, the value is set to $\beta=5$, but can be set by the station to suit their needs. If a block is not covered by the video logo, its counter is decremented by 1 to a minimum value of $\beta=0$. The final rating of a block is determined as:

$$
\operatorname{Rating}_{i}=\operatorname{Act}_{i}+\left(\operatorname{MaxAct}\left(\frac{\text { UsageCount }_{i}}{\text { MaxUsageCount }}\right)\right)
$$

where MaxAct is the maximum perceptual content activity value of all blocks, SsageCount $_{i}$ is the value of the counter for block $i$ and MaxU sageCount is the maximum counter value (in this case, MaxUsageCount $=5$ ). The video logo is placed at the location in the video broadcast where the block(s) have the lowest overall rating. By monitoring usage, it is possible to discourage the system from placing the video logo in the same place in consecutive shots.

\section{B. Logo Transparency Adjustment}

While adaptive video logo positioning is effective at preventing the video logo from obscuring perceptually important video broadcast content in many cases, there will still be situations where the video logo obscures important video broadcast content. For example, a scene may contain so much perceptual activity that even the area with least perceptual content activity contains important content. One approach for addressing such situations is to blend the logo with the video broadcast such that it appears semi-transparent. This allows content underneath the logo to be partially visible.

An issue with the use of video logos at a fixed transparency level is that the clarity of the video logo may be significantly reduced. This is particularly true if there is a low contrast between the logo and the underlying video broadcast content. For example, a predominately dark semi-transparent logo may become perceptually invisible if the underlying content is dark, making it very difficult to visibly watermark. However, it is important that the logo remains visible at all times. Therefore, the proposed algorithm addresses this issue by adaptively adjusting the transparency level of the video logo based on the contrast between the video logo and the underlying content. The logo transparency adjustment process is performed more frequently than the logo positioning process to ensure that the logo is visible at all times. In the proposed algorithm, the transparency level is re-calculated every 5 frames.

To determine the transparency level of the video logo, the average luminance of the video $\log L_{\text {logo }}$ and the average luminance of the underlying content in the video frame $L_{\text {content }}$ 
is first calculated in the range $[0,1]$. The transparency level (Trans) of the video logo is then determined based on the following:

$$
\text { Trans }=\text { Trans }_{\min }+\left(\text { Trans }_{\max }-\text { Trans }_{\min }\right)(|\Delta L|)
$$

where Trans $_{\max }$ and Trans $_{\min }$ are the maximum and minimum transparency levels respectively, and $\Delta L=$ $L_{\text {content }}-L_{\text {logo }}$. Therefore, transparency increases as the contrast between the video logo and the underlying content increases. This adaptive transparency adjustment helps ensure that the video logo remains visible at all times while allowing the underlying content to be partially visible through the logo.

\section{EXPERIMENTAL RESULTS}

It is difficult to establish a quantitative measure for evaluating the effectiveness of video logo placement. Measurements such as PSNR (Peak Signal-to-Noise Ratio) are meaningless in this context. For example, the positioning of a logo in the upper-right corner of a frame may result in the same PSNR loss as positioning the logo in the centre of a frame. However, the viewer will likely feel that the logo in the centre of a frame obscures more information.

To evaluate the effectiveness of the proposed algorithm, a video logo was embedded into four different test video streams from the Open Video Project [14] chosen at random. The perceptual quality of each video after logo placement was evaluated in a subjective manner based on two important properties:

1) Visibility of logo under various situations, and

2) Obstruction of perceptually important content.

Sample video frames are shown in Fig. 3. It is clear that the video logo does not obstruct any perceptually important video broadcast content. Also, the logo remains visible in both situations. Furthermore, the video logo in the first shot is at a different location than the second shot. This makes it more difficult to remove the video logo by simply cropping the video broadcast in a fixed manner. The experimental results demonstrate the effectiveness of the proposed algorithm in retaining logo clarity while reducing perceptual loss.

\section{CONCLUSIONS}

This paper presents a fast perceptually-adaptive video logo placement algorithm based on a perceptual model from both spatial and temporal perspectives. The algorithm adjusts the position and the transparency level of the video logo based on perceptual content activity, inter-frame relationships, and contrast. Experimental results demonstrate the effectiveness of the proposed scheme at achieving a good balance between perceptual quality and logo clarity. It is believed that this method can be used successfully for the placement of video logos in TV broadcasts.

\section{ACKNOWLEDGMENTS}

This research has been sponsored in part by Epson Canada and the Natural Sciences and Engineering Research Council of Canada.

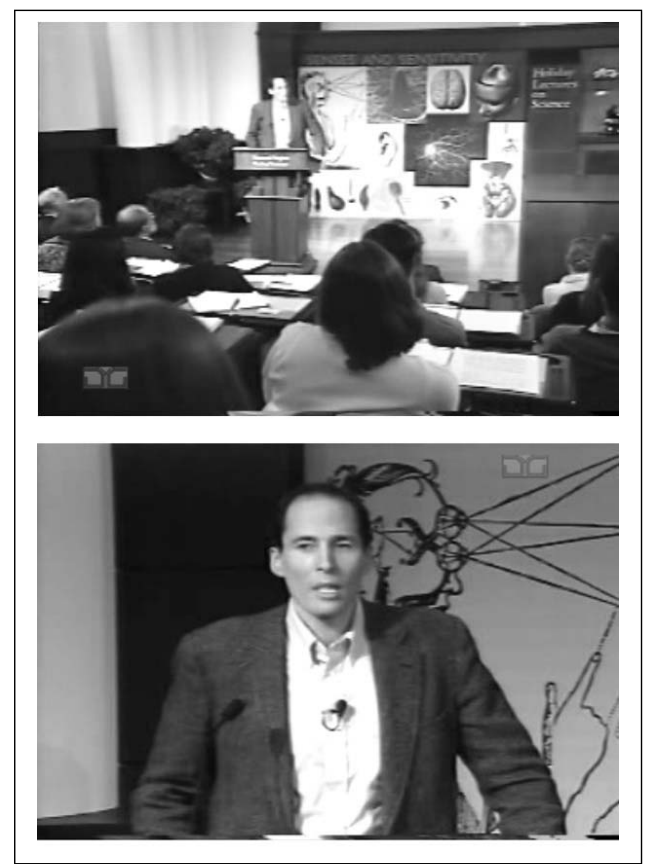

Fig. 3. Sample video frames from two different shots in a test video broadcast

\section{REFERENCES}

[1] S. Lee, Y. Suh, and Y. Ho, "Reversible image authentication based on watermarking," Proceedings of the IEEE International Conference on Multimedia and Expo, pp. 1321-1324, 2006.

[2] C. Podilchuk and W. Zeng, "Image-adaptive watermarking using visual models," IEEE Journal on Selected Areas in Communications, vol. 16, no. 4, pp. 525-539, 1998.

[3] D. Kundur and Dimitrios Hatzinakos, "Toward robust logo watermarking using multiresolution image fusion principles," IEEE Transactions on Multimedia, vol. 6, no. 1, pp. 185-198, 2004.

[4] B. Tao and B. Dickinson, "Adaptive watermarking in the DCT domain," Proceedings of the IEEE International Conference on Acoustics, Speech, and Signal Processing, pp. 2985-2988, 1997.

[5] S. Biswas, S. Das, E. Petriu, "An adaptive compressed MPEG-2 video watermarking scheme," IEEE Transactions on Instrumentation and Measurement, vol. 54, no. 5, pp. 1853-1861, 2005.

[6] C. Hzu and J. Wu, "Digital watermarking for video," Proceedings of the 13th International Conference on Digital Signal Processing, vol. 1, pp. 217-220, 1997.

[7] S. Yip, O. Au, C. Ho, H. Wong, "Lossless visible watermarking," Proceedings of the IEEE International Conference on Multimedia and Expo, pp. 853-856, 2006.

[8] J. Meng and S. Chang, "Embedding visible video watermarking in the compressed domain," Proceedings of the International Conference on Image Processing, pp. 474-477, 1998.

[9] S. Mohanty, K. Ramakrishnan, and M. Kankanhalli, "A DCT domain visible watermarking technique for images," Proceedings of the IEEE International Conference on Multimedia and Expo, pp. 1029-1032, 2000.

[10] M. Kankanhalli, Rajmohan, and K. R. Ramakrishnan, "Adaptive visible watermarking of images," Proceedings of the IEEE Conference on Multimedia Computing and Systems, vol. 1, pp. 568-573, 1999.

[11] Y. Wu, "Protection of video logos with randomization," Proceedings of the IEEE International Conference on Multimedia and Expo, pp. 1341$1344,2006$.

[12] A. Lumini and D. Maio, "Adaptive positioning of a visible watermark in a digital image," Proceedings of the IEEE International Conference on Multimedia and Expo, vol. 2, pp. 27-30, 2004.

[13] G. Langelaar, I. Setyawan, and R. Lagendijk, "Watermarking digital image and video data: A state-of-the-art overview," IEEE Signal Processing Magazine, vol. 17, no. 5, pp. 20-46, 2000.

[14] The Open Video Project, http://www.open-video.org, January 2007. 